\title{
Long-term outcomes in osteosarcoma patients in the Groote Schuur Hospital patient population: $A$ retrospective review
}

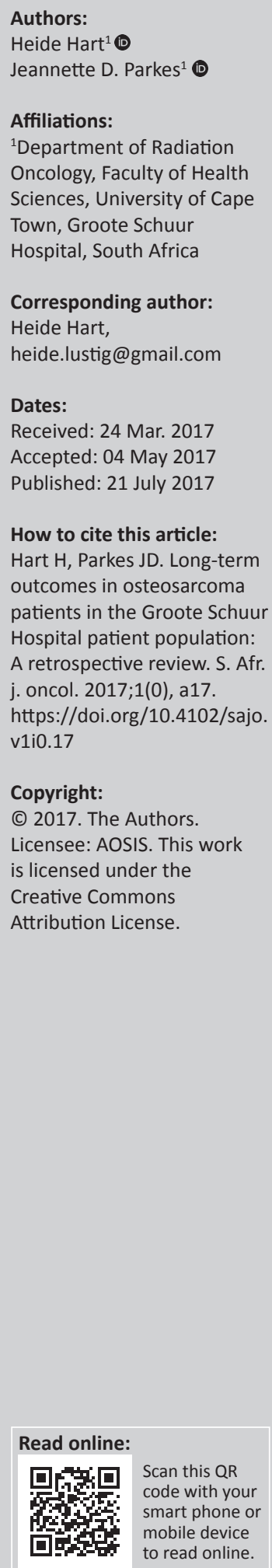

Background: Predictive factors for long-term outcomes in osteosarcoma patients are still controversial. There is no literature available regarding these factors in a patient population in a developing country.

Aim and setting: To determine the outcome of treatment of osteosarcoma patients treated at Groote Schuur Hospital from 1990 to 2012 in terms of locoregional control (LRC), disease-free survival (DFS) and overall survival (OS) and to determine the value of suggested predictive factors in this population.

Patients and methods: Retrospective review of all patients diagnosed with and treated for osteosarcoma at Groote Schuur Hospital between 1990 and 2012, considering OS, DFS and LRC. This review assesses the significance of suggested predictive factors from other studies, namely, HIV status, age at diagnosis, site of primary disease, type of chemotherapy used, response to chemotherapy and type of surgery in terms of OS, DFS and LRC.

Results: Forty-three patients with histologically confirmed osteosarcoma were treated at Groote Schuur Hospital between 1990 and 2012. Median 5 year OS was 57.8\%. On univariate analysis, the site of disease was the only statistically significant predictive factor for prognosis.

Conclusion: On univariate analysis, patients with axial disease have a worse predicted prognosis than those with primary disease in their extremities. The clinical behaviour and long-term outcome after treatment of these patients with osteosarcoma are similar to that seen internationally.

\section{Introduction}

Osteosarcomas are uncommon tumours, but they are the commonest primary bone tumour diagnosed, with a bimodal age distribution. A peak incidence occurs in childhood and adolescence and another peak occurs in elderly patients. ${ }^{1}$ The majority of osteosarcomas are found in the long bones of the extremities, but they can also be found in the axial skeleton and extraskeletally. ${ }^{2}$ Since the advent of adjuvant chemotherapy in the mid-1970s and more recently of neoadjuvant chemotherapy, there has been an improved overall survival (OS) seen in these patients. Since the 1980s, international data suggests 5-year OS rates of between $60 \%$ and $70 \%$ in patients with non-metastatic osteosarcoma of the extremities. ${ }^{3}$ Standard of care for osteosarcoma at present includes multi-drug chemotherapy followed by surgery and adjuvant chemotherapy. Radiotherapy is only used for irresectable or incompletely resected disease. ${ }^{2}$ Many studies have looked at predictive factors for prognosis, but the results remain conflicted. The suggested factors include: age at diagnosis, site of primary disease, type of surgery and response to neoadjuvant chemotherapy. ${ }^{4,5,6,7,8,9}$ Very little is available in the literature on HIV status and its effect on long-term outcomes in these patients. ${ }^{10}$

We do not have accurate data on the long-term outcome of osteosarcoma patients in South Africa and no data has previously been collected locally regarding predictive factors for prognosis.

We aimed to collect South African demographic data on osteosarcoma, including predictive factors. Furthermore, we aimed to establish whether our patients have similar characteristics to the available international data.

\section{Research methods and design}

The study is a retrospective review of the records of osteosarcoma patients at Groote Schuur Hospital who underwent treatment between 1 January 1990 and 31 December 2012. 
All osteosarcoma patients treated at Groote Schuur Hospital between 1990 and 2012 were included in the study.

Eligible patient records were identified using the electronic patient registry at Groote Schuur Hospital. A total of 42 patients were included in the initial analysis, but 5 were excluded because of defaulting any treatment other than their initial visit, leaving the remaining 37 patients as the patient population included in this case series. Of note, patients under 13 years of age were not included in this retrospective review, as they were treated at the children's hospital and not at Groote Schuur Hospital.

Patient demographics, treatment and follow-up data were collected and this included: age at diagnosis, race, gender, HIV status at diagnosis, date of diagnosis, site of primary tumour, metastatic status, chemotherapy received, date of surgery, type of surgery and histologic response to chemotherapy. Tumour necrosis of $90 \%$ or more was used as the cut-off value, based on the Huvos grading system to assess the pathological response to chemotherapy. ${ }^{2}$ The primary tumour size was not documented and could not be reliably documented. Other data collected included follow-up imaging and date of development of recurrence or metastases.

The primary endpoint of the study was to determine the outcome of treatment of osteosarcoma patients treated at Groote Schuur Hospital from 1990 to 2012 in terms of locoregional control (LRC), disease-free survival (DFS) and OS and to determine the value of suggested predictive factors in this population.

\section{Data analysis}

OS (defined in this review as time of diagnosis to time of death), LRC and DFS (both defined as the time from surgery to either local recurrence or diagnosis of metastases) were determined. These data were depicted on Kaplan-Meier curves. The patients lost to follow-up and those who survived without disease recurrence were censored. To compare groups, the log-rank test was used, with values less than $p=0.05$ being considered statistically significant. The data from each predictive factor were analysed according to the above stated methods. Only univariate analysis was performed because of small patient numbers.

\section{Ethical consideration}

The Human Research Ethics Committee of the University of Cape Town approved the conduct of this study (HREC/REF 413/2014).

\section{Results}

The number of patients included in this case series was 37 . Metastatic disease at presentation was present in eight $(21.6 \%)$ patients. Inoperable non-metastatic disease was present in four $(10.8 \%)$ patients at presentation. Twenty-five $(67.5 \%)$ patients were treated with radical intent.

The 5-year OS for the total population $(n=37)$ was $57.8 \%$ (95\% CI 31.7\% - 76.9\%), with the median OS not yet reached at the time of analysis. The 5-year LRC rate $(n=25)$ was $75.8 \%$ (95\% CI 50.5\% - 89.3\%) (Figure 1).

The 5-year disease-free-survival $(n=25)$ was $48.165 \%$ (95\% CI $25.2 \%-67.9 \%)$.

\section{Patient characteristics}

The demographic data of the study population is included in Table 1 . At presentation $30(81 \%)$ patients were performance status 1, 2(5\%) patients were performance status 2 and 1 patient was performance status 3-4. For four patients, performance status was not documented. The median time of follow-up for the patient population was 39 months.

\section{HIV status}

Of the four patients who were HIV positive at diagnosis, two had irresectable pulmonary metastatic disease upfront and received palliative chemotherapy, another one developed

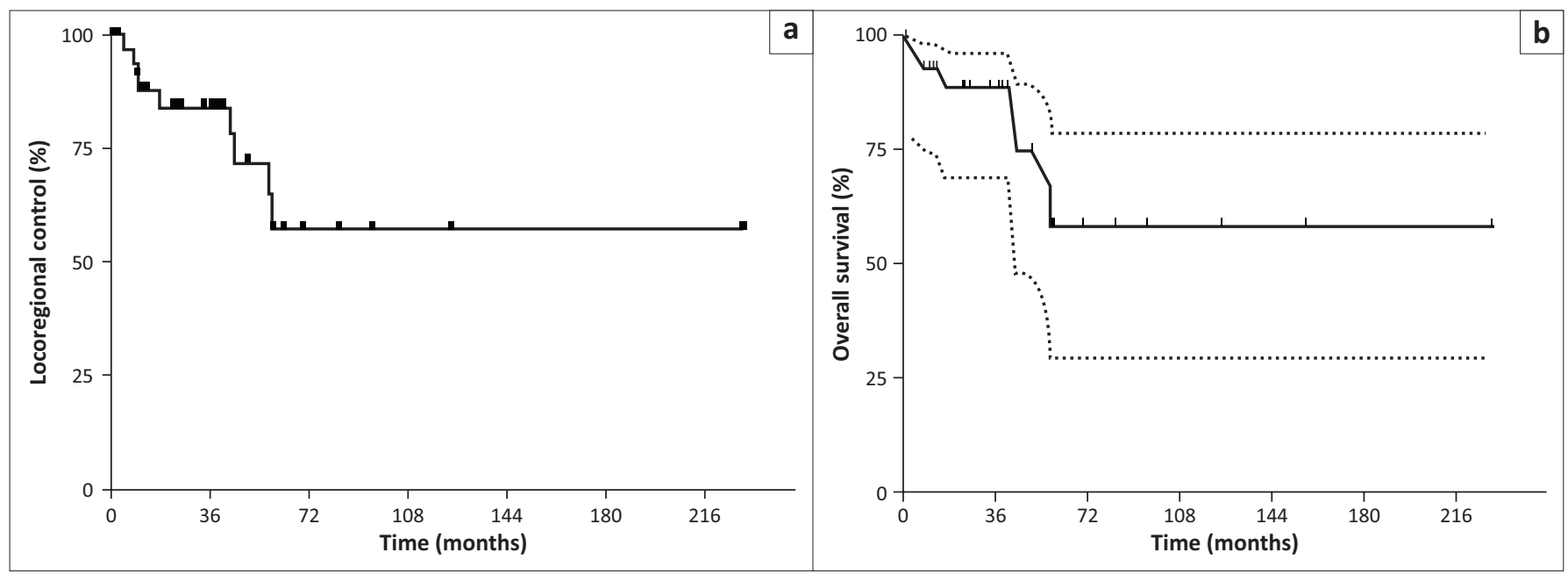

Recurrent or metastatic disease was detected on imaging and clinical examination. No histological confirmation was obtained in the majority of cases.

FIGURE 1: Kaplan-Meier survival curves for total population: LRC and OS (includes error bars which indicates small patient numbers leading to a large difference between results) in non-metastatic patients. 
TABLE 1: Demographic data of study population.

\begin{tabular}{|c|c|c|c|c|c|c|}
\hline Variable & $n$ Value & Percentage (\%) & Range & Median & Mean & Ratio \\
\hline Gender & & - & - & - & - & \\
\hline Male & $n=20$ & & & & & $1.18: 1$ \\
\hline Female & $n=17$ & & & & & \\
\hline Age & - & - & & & & - \\
\hline Male & & & $14-58$ years & 23 years & 28 years & \\
\hline Female & & & $13-85$ years & 21 years & 26 years & \\
\hline Total population & & & $13-85$ years & 23 years & 27 years & \\
\hline Race & & & - & - & - & - \\
\hline African & $n=11$ & 29.7 & & & & \\
\hline Caucasian & $n=12$ & 32.4 & & & & \\
\hline Coloured & $n=14$ & 37.8 & & & & \\
\hline HIV status at presentation & & & - & - & - & - \\
\hline Positive & $n=4$ & 11.0 & & & & \\
\hline Negative & $n=11$ & 30.0 & & & & \\
\hline Unknown & $n=22$ & 59.0 & & & & \\
\hline Metastatic disease at presentation & & & - & - & - & - \\
\hline Yes & $n=8$ & 21.6 & & & & \\
\hline No & $n=25$ & 67.5 & & & & \\
\hline No, but inoperable primary & $n=4$ & 10.8 & & & & \\
\hline Site of primary disease & & & - & - & - & - \\
\hline Trunk & $n=4$ & 10.8 & & & & \\
\hline Pelvis & $n=2$ & 5.4 & & & & \\
\hline Upper long bones & $n=5$ & 13.5 & & & & \\
\hline Lower long bones & $n=14$ & 40.0 & & & & \\
\hline Lower short bones & $n=12$ & 32.4 & & & & \\
\hline Histology & & & - & - & - & - \\
\hline Conventional subtype & $n=15$ & 54.0 & & & & \\
\hline Osteoblastic & $n=8$ & 40.5 & & & & \\
\hline Chondroblastic & $n=5$ & 5.0 & & & & \\
\hline Fibroblastic & $n=1$ & & & & & \\
\hline Telangiectatic & $n=1$ & & & & & \\
\hline Surface osteosarcomas & $n=2$ & & & & & \\
\hline Periosteal & $n=1$ & & & & & \\
\hline Parosteal & $n=1$ & & & & & \\
\hline Osteosarcoma, NOS & $n=20$ & & & & & \\
\hline Type of surgery performed & & & - & - & - & - \\
\hline Amputation & $n=13$ & 35.0 & & & & \\
\hline Limb sparing surgery & $n=16$ & 43.0 & & & & \\
\hline Unknown or no surgery & $n=8$ & 21.6 & & & & \\
\hline
\end{tabular}

NOS, not yet specified.

severe neutropenic sepsis on second line chemotherapy and opted to discontinue treatment and one patient was well when lost to follow-up.

\section{Site of primary disease}

The patients who had primary disease in the axial skeleton did worse than patients who had primary disease in the extremities. This was statistically significant for LRC, DFS and OS. Due to limited patient numbers in this cohort of patients, the 5-year survival rates according to the site of a patient's disease could only be commented on for the patients with pelvic and extremity primary site of disease. At 5 years, $0 \%$ of patients with pelvic primaries were alive, $75 \%$ of the patients with upper long bone primary disease, $70 \%$ of patients with lower short bone primary disease and $90 \%$ of patients with lower long bone primary disease. Patients with their primary tumour in the pelvis had a median OS of 17 months, those with a primary elsewhere in the trunk, 25.5 months and those with their primary in the lower limb short bones (defined as the tibia, the fibula and bones of the ankle and foot) had a median OS of 57 months. The patients who had primary tumours in the upper limb extremities (in this case series only in the humerus) or in the lower limb long bones (including the femur only) therefore had the best median OS which in this study had not yet been reached at the time of analysis $(p=0.0261)$. These results are reflected in the DFS and LRC where patients who had axial primaries had worse long-term outcomes in comparison to patients with an extremity primary.

\section{Chemotherapy and response to chemotherapy}

The majority of patients received multimodality treatment (see Figure 2). Patients who were deemed non-metastatic at presentation either received neoadjuvant chemotherapy followed by surgery and adjuvant chemotherapy or they had surgery prior to presentation to the Department of Radiation Oncology and received adjuvant chemotherapy. The majority of patients received a dual-drug chemotherapy regime of cisplatin $100 \mathrm{mg} / \mathrm{m}^{2} \mathrm{D} 1$ and adriamycin $25 \mathrm{mg} / \mathrm{m}^{2}$ 


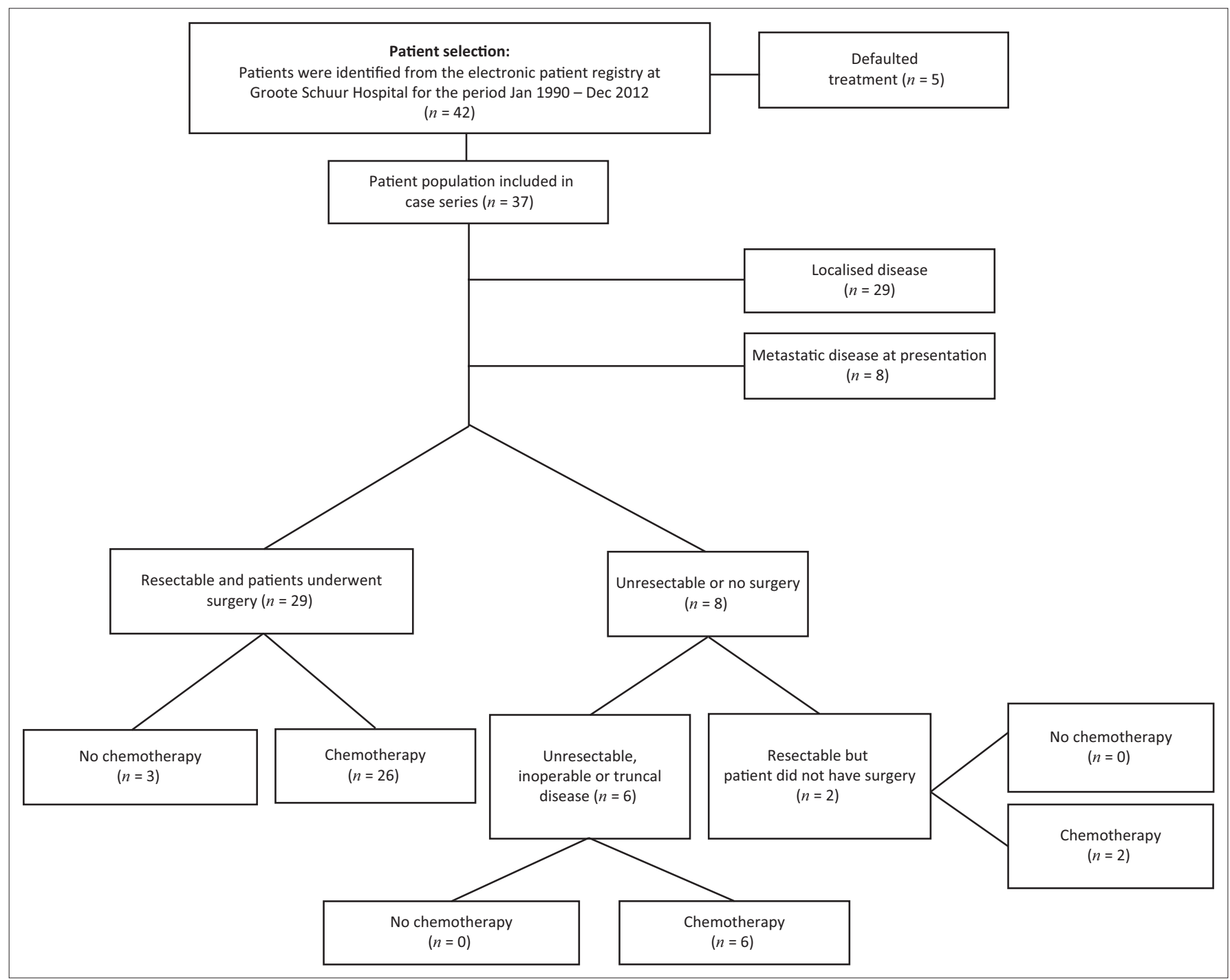

FIGURE 2: Flow diagram depicting patient identification, presentation and treatment.

D1-3. Of the 37 patients analysed, 34 patients received chemotherapy. Twenty-six patients received cisplatinadriamycin neoadjuvantly, seven patients received adjuvant chemotherapy only and one patient received high dose methotrexate (HDMTX) alternating with cisplatin and adriamycin. All long-term outcome results obtained from this analysis were not statistically significant and were difficult to interpret, because of the small patient numbers and the diversity of chemotherapy regimens used.

In terms of histological response to chemotherapy, 19 patients had their response assessed after receiving three cycles of neoadjuvant cisplatin-adriamycin; $21 \%$ of patients had a good response, with necrosis $\geq 90 \%$ and $79 \%$ had a poor response, with necrosis $<90 \%$. Amongst the patients who had a poor response, 1 was lost to follow-up post-surgery, 10 completed another two cycles of the cisplatin-adriamycin regimen followed by a change in chemotherapy with two cycles high dose methotrexate. Four patients completed another three cycles of the cisplatin-adriamycin regimen and did not change chemotherapy. Long-term outcome results for poorly responding patients whose chemotherapy regimens were changed, was not statistically significant: the median LRC time was not yet reached at the time of analysis for both groups ( $p=0.8203$ ) and the median DFS for the chemotherapy changed arm was 72.5 months versus 41.5 months for the chemotherapy unchanged arm $(p=0.8064)$. The median OS was not reached at the time of analysis for both arms $(p=0.8268)$.

\section{Discussion}

According to the SEER Program, osteosarcomas present with a bimodal age distribution, with a peak between 15 and 25 years of age and another later in life (> 60 years). ${ }^{1}$ This patient population had the highest incidence in patients younger than 25 years, in the 13-25-year age group (see Table 1). There was one patient over the age of 60 years. A specific analysis including age at diagnosis as a potential predictive factor was therefore not included in this article. The most common histological subtype of osteosarcoma diagnosed (90\% of patients), according to the World Health Organization, is the conventional subtype. ${ }^{11}$ However, the majority of patients were not sub-classified on initial histology report and have therefore been included in the series as 'osteosarcoma not otherwise specified' (Osteosarcoma, NOS). We have not included a discussion on stage of disease at 
diagnosis, as patients were classified as 'non-metastatic' versus 'metastatic' and 'operable' versus 'irresectable'.

The 5-year OS rates were similar to patients in international studies that received the two-drug regimen of cisplatin and adriamycin. ${ }^{12}$ The 5 -year LRC rate amongst our patients was high compared to the disease-free and OS rates and this could be attributed to a higher amputation rate than seen internationally. The higher amputation rate may indicate a higher proportion of patients presenting with locally advanced disease.

According to international data, the 5-year DFS and OS rates are not very different, reflecting that second line therapies are not generally found to be effective. ${ }^{3,13}$ In this study, a combination of ifosfamide and etoposide is used at recurrence. In this series, a difference was shown between the DFS and the OS which would indicate successful salvage treatment. However, it is worth noting that this series includes a small group of three patients who had indeterminate lung nodules at diagnosis which were assumed to be metastases.

Most of the patients included in this case series received three cycles of neo-adjuvant cisplatin-adriamycin, followed by either another three cycles of cisplatin-adriamycin after surgery or in the case of some of the younger poor responders, two cycles of cisplatin-adriamycin followed by two cycles of high dose methotrexate. In the poorly responding patients $(n=15)$, with necrosis $<90 \%$, there was no statistically significant difference in the long-term outcome of patients who changed regimen when compared to patients who continued with their chemotherapy unchanged. This has been shown in international literature too. Historically, it was thought that there might be some benefit, in changing chemotherapy post-operatively in younger poorly responding patients ( $<40$ years). However, in the recent results presented at EMSOS of the phase III randomisedcontrolled trial, EURAMOS1, results indicate that there is no benefit to changing the chemotherapy regimen in poorly responding patients and that it may be more toxic to do so. It is worth noting that, unlike our patients who were treated with cisplatin and adriamycin alone upfront, all patients in this trial received methotrexate, cisplatin and adriamycin with or without interferon.,14

The site of primary disease varies in different age groups. This was not apparent in our patient population, probably because of our limited number of patients and the fact that the majority of our patients were in the $<25$ year old age group. It has been shown that patients with their primary disease in the short bones of the upper limbs have the best prognosis, followed by disease of the other bones of the extremities. ${ }^{1,9,11}$ This is reflected in our results.

Patients who had limb sparing surgery had a more favourable long-term outcome when compared to patients who had amputations. However, there is a selection bias in this patient population because of large and locally advanced tumours preferentially requiring amputation. Patients with neurovascular involvement, rapidly growing tumours on neoadjuvant chemotherapy and skin involvement by tumour are not good candidates for limb sparing surgery. The number of limb sparing surgeries compared to amputations varies from centre to centre as better imaging and greater surgical expertise is required to perform the former.

Higher rates of limb sparing surgery are possible with timeous referral of patients. The rate of limb sparing surgery in the Western Cape is approximately $82 \%$. This correlates favourably with international figures. ${ }^{15}$

There is very limited data available on the effect of HIV status on prevalence and outcome in osteosarcoma. The data we collected were not complete for our entire case series, but we had the results of 18 patients, which is more than has been described in the literature to date. In South Africa, there is a national prevalence of HIV infection in adults of approximately $10.6 \%$, which is consistent with our patient population. ${ }^{10}$ Despite a trend towards HIV positive patients doing worse, there was no statistically significant difference in long-term outcome in the HIV positive or negative groups in our retrospective audit. Further research is required to determine an accurate relationship between osteosarcoma and HIV infection, as our patient numbers were small.

\section{Conclusion}

Osteosarcoma is a relatively rare cancer in which treatment has not changed significantly over the past 30 years. In our local setting, long-term outcome correlates with that found in the international literature. The site of a patient's primary disease has been found to be a significant predictive factor for OS overall and DFS in all patients. Further research needs to be conducted into other predictive factors as the patient numbers in our case series were limited. This case series is also a retrospective review in a single institution, which may have contributed to bias in the patients' results.

\section{Acknowledgements}

The authors would like to acknowledge and thank the following: Prof. Jeannette Parkes, Dr Alistair Hunter and Dr Keith Hoskings.

\section{Competing interests}

The authors declare that they have no financial or personal relationships that may have inappropriately influenced them in writing this article.

\section{Authors' contributions}

Data collection, analysis and manuscript composition was done by H.H. Senior review, expert consultation and final documentation approval was done by J.D.P. The authors alone are responsible for the content and writing of this article. 


\section{References}

1. Mirabello L, Troisi RJ, Savage SA. Osteosarcoma incidence and survival rates from 1973 to 2004: Data from the surveillance, epidemiology, and end results. Program. Cancer. 2009;115(7):1531-1543. https://doi.org/10.1002/cncr.24121

2. Xing D, Qasem SA, Owusu K, Zhang K, Siegal GP, Wei S. Changing prognostic factors in osteosarcoma: Analysis of 381 cases from two institutions. Hum Pathol. 2014;45(8):1688-1696. https://doi.org/10.1016/j.humpath.2014.04.010

3. Luetke A, Meyers PA, Lewis I, Juergens H. Osteosarcoma treatment - Where do we stand? A state of the art review. Cancer Treat Rev. 2014;40(4):523-532. https:// doi.org/10.1016/j.ctrv.2013.11.006

4. Hartmann JT, Kopp H-G. Bone sarcomas. Update Canc Therapeut. 2006;1(1) 65-74. https://doi.org/10.1016/j.uct.2006.04.008

5. Wang LL, Chintagumpala M, Gebhardt MC. Osteosarcoma: Epidemiology, pathogenesis, clinical presentation, diagnosis and histology [homepage on the Internet]. 2014 [cited 2014 May 25]. Available from: http://www.uptodate.com

6. Harting MT, Lally KP, Andrassy RJ, Vaporciyan AA, Cox CS, Jr., Hayes-Jordan A, et al. Age as a prognostic factor for patients with osteosarcoma: An analysis of 438 patients. J Cancer Res Clin Oncol. 2010;136(4):561-570. https://doi.org/10.1007/ s00432-009-0690-5

7. Janeway KA, Goorin AM, Maki R. Chemotherapy and radiation therapy in the management of osteosarcoma [homepage on the Internet]. 2014 [cited 2014 May 25]. Available from: http://www.uptodate.com

8. Bacci G, Mercuri M, Longhi A, Ferrari S, Bertoni F, Versari M, et al. Grade of chemotherapy-induced necrosis as a predictor of local and systemic control in 881 patients with non-metastatic osteosarcoma of the extremities treated with neoadjuvant chemotherapy in a single institution. Eur J Cancer. 2005;41(14): 2079-2085. https://doi.org/10.1016/j.ejca.2005.03.036

9. Bacci $G$, Longhi A, Versari M, Mercuri M, Briccoli A, Picci P. Prognostic factors for osteosarcoma of the extremity treated with neoadjuvant chemotherapy: 15-year experience in 789 patients treated at a single institution. Cancer. 2006;106(5):1154-1161. https://doi.org/10.1002/cncr.21724

10. Marais LC, Ferreira N. Osteosarcoma in adult patients living with HIV/AIDS. ISRN Oncol. 2013;2013:219369.

11. Rosenburg AE, Cleton-Jansen A-M, De Pinieux $G$, et al. Conventional osteosarcoma. In: Fletcher CDM, Bridge JA, Hogendoorn PCW, Mertens F, editors. WHO classification of tumours of soft tissue and bone. 4th ed. Lyon: IARC, 2013; p. 282.

12. Souhami RL, Craft AW, Van der Eijken JW, et al. Randomised trial of two regimens of chemotherapy in operable osteosarcoma: A study of the European Osteosarcoma Intergroup. Lancet. 1997;350(9082):911-917. https://doi.org/10.1016/S01406736(97)02307-6

13. Gelderblom H, Jinks RC, Sydes $M$, et al. Survival after recurrent osteosarcoma: Data from 3 European Osteosarcoma Intergroup (EOI) randomized controlled trials. Eur J Cancer. 2011;47(6):895-902. https://doi.org/10.1016/j.ejca.2010. 11.036

14. Whelan JS, Bielack S, Smeland S, et al. EURAMOS-1: Results from the randomised questions of the first European-American osteosarcoma study. Abstract. 28th Annual Meeting of the European Musculo-Skeletal Oncology Society (EMSOS); Athens, Greece; 29 April-1 May 2015.

15. Laubscher M, Hoffman EB, Hosking KV Osteosarcoma in children and adolescents: A 20 year study. Proceedings: 56th South African Association Orthopedic Congress; Pretoria, South Africa; Sept 2010. 